\title{
Rayleigh wave dispersion equation with real terms in a layered transversely isotropic half space
}

\author{
Sankar N. Bhattacharya ${ }^{1, *}$ \\ ${ }^{1}$ Lithosphere Study Centre, New Delhi, India
}

Article history

Received May 14, 2017; accepted November 12, 2017.

Subject classification:

Seismic anisotropy; Rayleigh waves; Computational seismology; Wave propagation; Theoretical seismology.

\begin{abstract}
We consider transversely isotropic media with vertical axis of symmetry (VTI). Solutions of P-SV equation of motion in a homogeneous VTI medium contain depth decay factors $r_{1}$ and $r_{2}$, which sometimes become complex depending on medium parameters. In this case, $r_{1}$ and $r_{2}$ are complex conjugates. Using this property, we obtain dispersion equation of Rayleigh waves with real terms for a layered VTI half space through Thomson-Haskell method with reduced delta matrix. Phase and group velocities as well as surface ellipticity of Rayleigh waves are computed in real domain for a few oceanic and continental VTI structures of the earth. Present computation in real domain is similar to that in a layered isotropic half space using the same method. Thus it is presumed that such computation in a layered VTI half space will allow efficient evaluation of a VTI structure of the earth.
\end{abstract}

\section{Introduction}

Transverse isotropy with vertical axis of symmetry (VTI) or radial anisotropy of the crust and upper mantle has been noted through the incompatibility to fit Rayleigh and Love wave dispersion with an isotropic structure [Anderson 1961]. During last few decades this incompatibility shows the variation of VTI in the upper mantle of various regions [Kustowski et al. 2008] indicating mantle flow and discriminating different geodynamic processes and thermo-chemical convective models [Auer et al. 2015, Khan et al. 2011]. VTI in the crust has also been seen in several regions [Shapiro et al. 2004, Raykova and Nikolova 2003, Moschetti et al. 2010]. In exploration seismology, better seismic images using reflection data are obtained with VTI in the subsurface [Thomsen 1986, Alkalifah and Tsvankin 1995].

The estimation of underground structure using surface waves is based on an inversion in which observed surface wave dispersion is compared with theoretical one for an assumed structure usually with a horizontally layered medium. In an isotropic layered half space, computation of theoretical surface wave dispersion by Thomson-Haskell matrix method or T-H method [Haskell 1953] has proven to be a powerful tool. The restatement of recursive relation in terms of delta matrix (also called compound matrix or second order sub-determent) is a significant improvement in T-H method [Dunkin 1965, Thrower 1965]. This was further improved by reduced delta matrix [Watson 1970]. Stability of T-H method at high frequencies can be improved by reduction of deeper layers, whose contributions to dispersion are negligible [Bhattacharya 1987]. The reflection-transmission method in a layered isotropic half space is a complex arithmetic scheme proposed by Kennett [1974] which is stable at very high frequencies. At present it has been developed as a generalized reflection-transmission coefficient method, or, in brief RT method [Kennett 1983, Luco and Aspel 1983, Chen 1993]. However, this method is least efficient of all improved methods [Buchen and BenHardor 1997]; Pei et al. [2008] improved this method to increase the speed of computation. Efficiency of this method is low because of complex arithmetic and dispersion equations consist of real and imaginary parts, both of which are to be considered during root finding.

As indicated in the next section, solutions of P-SV equations in a homogeneous VTI layer contain $\exp \left( \pm k r_{j} z\right)$, where $z$ is the vertical coordinate, $k$ is wave number and $j=1,2 ; r_{j}$ may be considered as depthdecay factor. Rayleigh wave dispersion equation in a layered VTI half space becomes complex when $r_{j}$ becomes complex in one or more layers [Anderson 1961, 1965]. In such a layered half space, Anderson [1961] and Harkrider and Anderson [1962] computed surface wave dispersion in a layered VTI half space by extending the T-H method. Harkrider and Anderson [1962] replaced complex $r_{j}$ in each layer by one from 
equivalent isotropic layer to avoid the computation in a complex domain. The method of Anderson [1961] has been improved by doubled T-H method [Ke et al. 2011] and also by delta-matrix method [Ikeda and Matsuoka 2013]. In both the studies, since $r_{j}$ becomes complex in some layers, complex numbers are used in the computations; as in RT method, roots need to be evaluated both from real and complex parts of the dispersion equation. For Love wave dispersion computation using T-H method in a layered VTI half space, there is no such problem and dispersion equation is obtained with real terms [Anderson 1962]. However, such dispersion computation for Rayleigh waves is being hampered due to complex terms in the dispersion equation. On the other hand, the T-H method for Rayleigh waves and its improvements [Buchen and Ben-Hardor 1997] in an isotropic medium are so popular that that it has been used even to evaluate radially anisotropic medium, where it is assumed that vertically and horizontally polarised $S$ wave velocities $\left(\beta_{\mathrm{V}}\right.$ and $\left.\beta_{\mathrm{H}}\right)$ are different but vertical and horizontal P-wave velocities $\left(\alpha_{\mathrm{V}}\right.$ and $\left.\alpha_{\mathrm{H}}\right)$ are equal; it is also assumed that the fifth parameter $\eta=1$ [Shapiro et al. 2004, Raykova and Nilolova 2003, Moschetti et al. 2010, Xie et al. 2013]. Xie et al. [2013] mentioned that this assumption is physically unrealistic in real assemblages, where $S$-wave anisotropy would be accompanied by P-wave anisotropy with $\eta$ differing from unity [Babuska and Cara 1991]. Further, Bhattacharya [2015] has shown that dispersion curves with such assumption for VTI structure are close to the correct result for Love waves; but such assumption is not satisfactory for Rayleigh waves. It has also been observed that this assumption overestimates S-wave anisotropy.

When $r_{j}$ is complex in a VTI medium, $r_{1}$ and $r_{2}$ are complex conjugates. Using this property, we get a layer matrix as well as a reduced delta layer matrix, where each element is real even if $r_{j}$ is complex in any layer. In a layered VTI half space, we obtain a Rayleigh wave dispersion equation which remains real with or without presence of complex $r_{j}$ in any layer. Dispersion curves as well as surface ellipticity are computed in real domain for oceanic and continental layered VTI half space.

\section{Basic equations}

Let us consider a homogeneous VTI medium with anisotropic parameters as $\alpha_{\mathrm{H}}, \beta_{\mathrm{V}}, \xi$, $\phi$ and $\eta$ [Takeuchi and Saito 1972, Nishimura and Forsyth 1989]. Solutions of such medium were obtained by Anderson [1961], who used elastic stiffness coefficients $c_{m n}$ as medium parameters [Love 1927]. These two representations of elastic parameters are related by $\alpha_{\mathrm{H}}^{2}\left[=c_{11} / \rho\right], \alpha_{\mathrm{V}}^{2}\left[=c_{33} / \rho\right]$, $\beta_{\mathrm{V}}^{2}\left[=c_{44} / \rho\right], \beta_{\mathrm{H}}^{2}\left[=\left(c_{11}{ }^{-} c_{12}\right) / 2 \rho\right]$ and $\eta\left[=c_{13} /\left(c_{11}-2 c_{44}\right)\right]$ [Takeuchi and Saito, 1972); $\xi=\left(\beta_{\mathrm{H}}^{2}\right) /\left(\beta_{\mathrm{V}}^{2}\right), \phi=\left(\alpha_{\mathrm{V}}^{2}\right)\left(\alpha_{\mathrm{H}}^{2}\right) ;$ $\xi$ and $\phi$ are $S$-wave and P-wave anisotropy respectively. Let the waves propagate in $x$-z plane where $x$ is along horizontal plane and $z$ is vertical (upward). Following Anderson [1961], the displacement components $u$ and $w$ along $x$ and $z$ axes are written as

$$
[u, w]=[\mathrm{U}(z), W(z)] \exp [i k(c t-x)]
$$

where $c$ is phase velocity, $k$ is the wave number and

$$
\begin{aligned}
& U(z)=A_{1} e^{k r_{1} z}+A_{2} e^{k r_{2} z}+B_{1} e^{-k r_{1} z}+B_{2} e^{-k r_{2} z} \\
& -i W(z)=\gamma_{1} A_{1} e^{k r_{1} z}+\gamma_{2} A_{2} e^{k r_{2} z}-\gamma_{1} B_{1} e^{-k r_{1} z}-\gamma_{2} B_{2} e^{-k r_{2} z}
\end{aligned}
$$

with

$$
\begin{gathered}
\gamma_{j}=\frac{r_{j} \tilde{G}}{\phi \alpha_{H}^{2} r_{i}^{2}-\beta_{V}^{2}+c^{2}}=-\frac{\beta_{V}^{2} r_{j}^{2}-\alpha_{H}^{2}+c^{2}}{r_{j} \tilde{G}} \\
r_{j}^{2}=\left(S_{1} \pm S_{3}\right) / 2, \quad j=1,2 \\
S_{1}=A+B-C, \quad S_{2}=A B, \quad S_{3}^{2}=S_{1}^{2}-4 S_{2} \\
A=\frac{1}{\beta_{V}^{2}}\left(\alpha_{H}^{2}-c^{2}\right), \quad B=\frac{1}{\phi \alpha_{H}^{2}}\left(\beta_{V}^{2}-c^{2}\right), \quad C=\frac{1}{\phi}\left[\frac{\eta\left(\alpha_{H}^{2}-2 \beta_{V}^{2}\right)+\beta_{V}^{2}}{\alpha_{H} \beta_{V}}\right]^{2} \\
\tilde{G}=\eta\left(\alpha_{H}^{2}-2 \beta_{V}^{2}\right)+\beta_{V}^{2}
\end{gathered}
$$

When $S_{3}^{2} \geq 0, r_{j}^{2}$ is real making $r_{j}$ to be either pure real or pure imaginary and is given by

$$
r_{j}=\frac{1}{\sqrt{ } 2} \sqrt{ }\left[S_{1} \pm S_{3}\right]
$$

When $S_{3}^{2}<0, r_{\mathrm{j}}^{2}$ is complex and given by

$$
r_{j}^{2}=\frac{1}{2}\left[S_{1} \pm i \sqrt{4 S_{2}-S_{1}^{2}}\right],
$$

As given in Equation (5), $S_{1}$ is real and so $r_{j}^{2}$ is complex only when $S_{3}^{2}<0$. Using (5), we can write

$$
S_{3}^{2}=(A-B)^{2}+C^{2}-2 C(A+B)
$$

Thus $S_{3}^{2}$ is negative only if $(A+B)>0$. From (5), $S_{2}=\left(S_{1}^{2}-S_{3}^{2}\right) / 4$. Thus $S_{2}$ is positive when $S_{3}^{2}$ is negative. Equation (5) shows that $S_{2}$ can be positive if $A$ and $B$ are either, both positive or, both negative. Thus to have $(A+B)>0$, both $A$ and $B$ will be positive and (5) shows that $c<\min \left[\alpha_{\mathrm{H}}, \beta_{\mathrm{V}}\right]$. Normally $\beta_{\mathrm{V}}<\alpha_{\mathrm{H}}$, therefore $c<\beta_{\mathrm{V}}$ when $S_{3}^{2}$ is negative.

$S_{3}^{2}$ is a nonlinear function of $c / \beta_{\mathrm{V}}, \alpha_{\mathrm{H}} / \beta_{\mathrm{V}}, \phi$ and $\eta$. In a $\phi-\eta$ plane the region of negative $S_{3}^{2}$ or complex $r_{j}^{2}$ lies between two nearly parallel lines (Figure 1). The $\phi$ and $\eta$ values above complex region are rarely observed. With normal values of $\phi$ and $\eta$ in the crust and mantle, complex $r_{j}^{2}$ exists when $\phi$ is low and $\eta$ is high. In the subsurface, $\phi$ may be very low and $\eta$ may be very high [Xiao 2004]. Strong VTI has often been noted in organic rich shales [Sengupta et al. 2015]. As $c / \beta_{\mathrm{V}}$ dcreases, the com- 


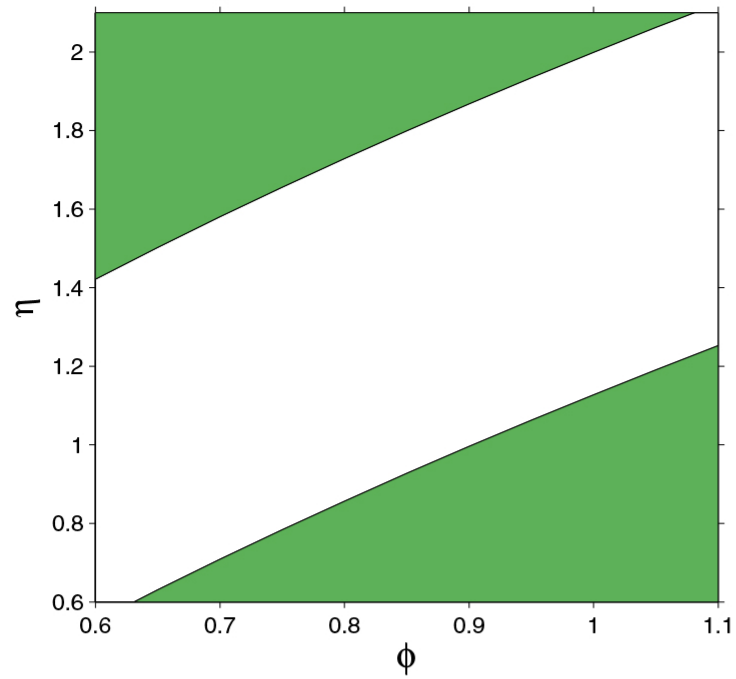

Figure 1. With $\alpha_{\mathrm{H}} / \beta_{\mathrm{V}}=1.732$ and $c / \beta_{\mathrm{V}}=0.90$, the green shaded region in $\phi-\eta$ plane shows $\phi$ and $\eta$ values for which $S_{3}^{2}$ is positive or $r_{\mathrm{j}}^{2}$ is real. White region shows $\phi$ and $\eta$ values for which $S_{3}^{2}$ is negative or $r_{j}^{2}$ is complex. $\phi$ and $\eta$ are anisotropic parameters [Takeuchi and Saito 1972, Nishimura and Forsyth 1989].

plex region spreads towards both lower and higher anisotropy. As $\alpha_{\mathrm{H}} / \beta_{\mathrm{V}}$ increases, the complex region is narrower with upper boundary coming down and lower boundary having a minor change.

(6)

When $S_{3}^{2}<0, r_{j}^{2}$ as well as $r_{j}$ is complex and from

$$
r_{j}=\frac{1}{2}\left\{\sqrt{ }\left[2 \sqrt{S_{2}}+S_{1}\right] \pm i \sqrt{ }\left[2 \sqrt{S_{2}}-S_{1}\right]\right\}=r^{\prime} \pm i r^{\prime \prime}
$$

showing $r_{1}$ and $r_{2}$ as complex conjugates. In (7a)

$$
r^{\prime}=\frac{1}{2} \sqrt{ }\left[2 \sqrt{S_{2}}+S_{1}\right] \text { and } r^{\prime \prime}=\frac{1}{2} \sqrt{[}\left[2 \sqrt{S_{2}}-S_{1}\right]
$$

Since $S_{3}^{2}<0$, (5) shows that $4 S_{2}>S_{1}^{2}$, or $2 \sqrt{ }\left(S_{2}\right)>S_{1}$, because both $S_{2}$ and $S_{1}^{2}$ are positive. Thus r' and r" are real and positive.

In isotropic case $\xi=\phi=\eta=1, \alpha_{\mathrm{H}}=\alpha_{\mathrm{V}}=\alpha$ and $\beta_{\mathrm{V}}=\beta_{\mathrm{H}}=\beta$. Thus

$A=\left(\alpha^{2}-c^{2}\right) / \beta^{2}, \mathrm{~B}=\left(\beta^{2}-c^{2}\right) / \alpha^{2}$ and $C=\alpha^{2} / \beta^{2}+\beta^{2} / \alpha^{2}-2$.

Thus

$$
\left(S_{1}, S_{3}\right)=\left(1-c^{2} / \alpha^{2}\right) \pm\left(1-c^{2} / \beta^{2}\right)
$$

and

$$
r_{1}=\sqrt{1-c^{2} / \alpha^{2}}=r_{\alpha} \text { and } r_{2}=\sqrt{1-c^{2} / \beta^{2}}=r_{\beta} .
$$

Further

$$
\gamma_{1}=r_{\alpha} \text { and } \gamma_{2}=1 / r_{\beta} \text {. }
$$

\section{Layer matrix}

\subsection{Formulation}

Here we find the layer matrix of a given layer [Haskell 1953]. The stress components $p_{z z}$ and $p_{z x}$ in terms of $U(z)$ and $W(z)$ are in Anderson [1961]. Thus displacement-stress matrix is written as

$$
[Y(z)]=[E(z)][R]
$$

where

$$
\begin{gathered}
{[Y(z)]=\left[U(z),-i W(z), i p_{z z} / k, p_{z x} / k\right]^{T}} \\
{[R]=\left[A_{1}+B_{1}, A_{1}-B_{1}, A_{2}+B_{2}, A_{2}-B_{2}\right]^{T}}
\end{gathered}
$$

and

$$
\begin{gathered}
{[E(z)]=\left[\begin{array}{cccc}
\cosh \bar{Q}_{1} & \sinh \bar{Q}_{1} & \cosh \bar{Q}_{2} & \sinh \bar{Q}_{2} \\
\gamma_{1} \sinh \bar{Q}_{1} & \gamma_{1} \cosh \bar{Q}_{1} & \gamma_{2} \sinh \bar{Q}_{2} & \gamma_{2} \cosh \bar{Q}_{2} \\
X_{1} \cosh \bar{Q}_{1} & X_{1} \sinh \bar{Q}_{1} & X_{2} \cosh \bar{Q}_{2} & X_{2} \sinh \bar{Q}_{2} \\
Z_{1} \sinh \bar{Q}_{1} & Z_{1} \cosh \bar{Q}_{1} & Z_{2} \sinh \bar{Q}_{2} & Z_{2} \cosh \bar{Q}_{2}
\end{array}\right]} \\
\bar{Q}_{j}=k r_{j} z, X_{j}=c_{13}-c_{33} r_{j} \gamma_{j}, Z_{j}=\left(r_{j}+\gamma_{j}\right) c_{44} \\
c_{13}=\eta \rho\left(\alpha_{H}^{2}-2 \beta_{V}^{2}\right), c_{33}=\rho \phi \alpha_{H}^{2}, c_{44}=\rho \beta_{V}^{2}
\end{gathered}
$$

Evaluating $Y(-H)$ and $Y(0)$ from (12) and eliminating $[R]$ we get

$$
[Y(-H)]=[E(-H)][E(0)]^{-1}[Y(0)]=[a][Y(0)]
$$

Here $H$ is the thickness of the layer, $[Y(-H)]$ is the displacement-stress matrix at the bottom of the layer and $[Y(0)]$ is that at the top. In (17), $[a]$ is the layer matrix given by

$$
[a]=[E(-H)][E(0)]^{-1}
$$

where from (14)

$$
\begin{gathered}
{[E(-H)]=\left[\begin{array}{cccc}
\cosh Q_{1} & -\sinh Q_{1} & \cosh Q_{2} & -\sinh Q_{2} \\
-\gamma_{1} \sinh Q_{1} & \gamma_{1} \cosh Q_{1} & -\gamma_{2} \sinh Q_{2} & \gamma_{2} \cosh Q_{2} \\
X_{1} \cosh Q_{1} & -X_{1} \sinh Q_{1} & X_{2} \cosh Q_{2} & -X_{2} \sinh Q_{2} \\
-Z_{1} \sinh Q_{1} & Z_{1} \cosh Q_{1} & -Z_{2} \sinh Q_{2} & Z_{2} \cosh Q_{2}
\end{array}\right]} \\
{[E(0)]^{-1}=\left[\begin{array}{cccc}
-X_{2} / X_{12} & 0 & 1 / X_{12} & 0 \\
0 & Z_{2} / \Gamma_{12} & 0 & -\gamma_{2} / \Gamma_{12} \\
X_{1} / X_{12} & 0 & -1 / X_{12} & 0 \\
0 & -Z_{1} / \Gamma_{12} & 0 & \gamma_{1} / \Gamma_{12}
\end{array}\right]} \\
X_{12}=X_{1}-X_{2} \text { and } \quad \Gamma_{12}=\gamma_{1} Z_{2}-\gamma_{2} Z_{1}
\end{gathered}
$$

Thus from (18) $[a]=$

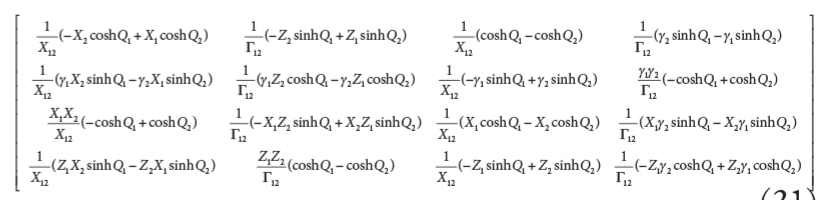




\subsection{Case I: $\mathrm{S}_{3}^{2} \geq 0$}

In this case, $r_{j}$ is either real or imaginary. We shall rearrange the matrix elements of $[a]$ in (21) so that each element becomes real even if $r_{i}$ is imaginary. Let us denote

$$
g_{j}=\frac{\tilde{G}}{\phi \alpha_{H}^{2} r_{j}^{2}-\beta_{V}^{2}+c^{2}}
$$

Thus from (3), $\gamma_{j}=r_{j} g_{j}$. From (15), we have

$X_{j}=c_{13}-c_{33} r_{j}^{2} g_{j}$ and $Z_{j}=c_{44} r_{j}\left(1+g_{j}\right)$

It may be further noted that

$$
\Gamma_{12}=c_{44} r_{1} r_{2}\left(g_{1}-g_{2}\right)=c_{44} r_{1} r_{2} g_{12}
$$

where $g_{12}=g_{1}-g_{2}$. Now we write the elements of layer matrix $[a]$ as

$$
\begin{gathered}
a_{11}=\frac{1}{X_{12}}\left(-X_{2} \cosh Q_{1}+X_{1} \cosh Q_{2}\right), \\
a_{12}=\frac{1}{g_{12}}\left[-\left(1+g_{2}\right) \frac{\sinh Q_{1}}{r_{1}}+\left(1+g_{1}\right) \frac{\sinh Q_{2}}{r_{2}}\right], \\
a_{13}=\frac{1}{X_{12}}\left(\cosh Q_{1}-\cosh Q_{2}\right), \\
a_{14}=\frac{1}{c_{44} g_{12}}\left[g_{2} \frac{\sinh Q_{1}}{r_{1}}-g_{1} \frac{\sinh Q_{2}}{r_{2}}\right], \\
a_{22}=\frac{1}{g_{12}}\left[g_{1}\left(1+g_{2}\right) \cosh Q_{1}-g_{2}\left(1+g_{1}\right) \cosh Q_{2}\right], \\
a_{23}=\frac{1}{X_{12}}\left(-g_{1} r_{1} \sinh Q_{1}-X_{1} g_{2} r_{2} \sinh Q_{2}\right), \\
a_{44}=\frac{1}{g_{12}}\left[-g_{2}\left(1+g_{1}\right) \sinh Q_{2}\right), \\
a_{24}=\frac{g_{1} g_{2}}{c_{44} g_{12}}\left(-\cosh Q_{1}+\cosh Q_{2}\right), \\
\left.a_{42}=\frac{c_{44}}{X_{12}}\left[X_{2}\left(1+g_{12}\right) r_{1} \sinh Q_{1}-X_{1}\left(1+g_{2}\right) r_{2} \sinh Q_{2}\right], \cosh Q_{2}\right] . \\
a_{31}=\frac{x_{1} X_{2}}{X_{12}}\left(-\cosh Q_{1}+\cosh Q_{2}\right), \\
a_{32}=\frac{1}{g_{12}}\left[-X_{1}\left(1+g_{2}\right) \frac{\sinh Q_{1}}{r_{1}}+X_{2}\left(1+g_{1}\right) \frac{\sinh Q_{2}}{r_{2}}\right], \\
a_{33}=\frac{1}{x_{12}}\left(X_{1} \cosh Q_{1}-X_{2} \cosh Q_{2}\right),
\end{gathered}
$$

Similar elements were also derived by Harkrider and Anderson [1962]. Note that $\cosh Q_{j},\left(\sinh Q_{j}\right) / r_{j}$ and $r_{j} \sinh Q_{j}$ are real when $r_{j}$ is imaginary.

\subsection{Case II: $S_{3}^{2}<0$}

In this case $r_{1}$ and $r_{2}$ are complex conjugates and so $Q_{1}$ and $Q_{2}$ are also complex conjugates and we denote

$$
Q_{1}=Q^{\prime}+i Q^{\prime \prime} \text { and } Q_{2}=Q^{\prime}-i Q^{\prime \prime}
$$

where $Q^{\prime}=k r^{\prime} H$ and $Q^{\prime \prime}=k r^{\prime \prime} H$. Substituting in (21) and denoting

$$
\begin{array}{ll}
F_{1}=\cosh Q^{\prime} \cos Q^{\prime \prime}, & F_{2}=\cosh Q^{\prime} \sin Q^{\prime \prime}, \\
F_{3}=\sinh Q^{\prime} \sin Q^{\prime \prime}, & F_{4}=\sinh Q^{\prime} \cos Q^{\prime \prime},
\end{array}
$$

we get the elements of the layer matrix as

$$
\begin{aligned}
& a_{11}=F_{1}-i \frac{X_{1}+X_{2}}{X_{12}} F_{3}, \\
& a_{12}=-i \frac{Z_{1}+Z_{2}}{\Gamma_{12}} F_{2}+\frac{Z_{1}-Z_{2}}{\Gamma_{12}} F_{4}, \\
& a_{13}=i \frac{2}{X_{12}} F_{3}, \\
& a_{14}=i \frac{\gamma_{1}+\gamma_{2}}{\Gamma_{12}} F_{2}-\frac{\gamma_{1}-\gamma_{2}}{\Gamma_{12}} F_{4}, \\
& a_{21}=i \frac{X_{1} \gamma_{2}+X_{2} \gamma_{1}}{X_{12}} F_{2}-\frac{X_{1} \gamma_{2}-X_{2} \gamma_{1}}{X_{12}} F_{4}, \\
& a_{22}=F_{1}+i \frac{\gamma_{1} Z_{2}+\gamma_{2} Z_{1}}{\Gamma_{12}} F_{3}, \\
& a_{23}=-i \frac{\gamma_{1}+\gamma_{2}}{X_{12}} F_{2}-\frac{\gamma_{1}-\gamma_{2}}{X_{12}} F_{4}, \\
& a_{24}=-i \frac{2 \gamma_{1} \gamma_{2}}{\Gamma_{12}} F_{3}, \\
& a_{31}=-i \frac{2 X_{1} X_{2}}{X_{12}} F_{3}, \\
& a_{32}=-i \frac{X_{1} Z_{2}+X_{2} Z_{1}}{\Gamma_{12}} F_{2}-\frac{X_{1} Z_{2}-X_{2} Z_{1}}{\Gamma_{12}} F_{4}, \\
& a_{33}=F_{1}+i \frac{X_{1}+X_{2}}{X_{12}} F_{3}, \\
& a_{34}=i \frac{X_{1} \gamma_{2}+X_{2} \gamma_{1}}{\Gamma_{12}} F_{2}+\frac{X_{1} \gamma_{2}-X_{2} \gamma_{1}}{\Gamma_{12}} F_{4} \\
& a_{41}=i \frac{X_{1} Z_{2}+X_{2} Z_{1}}{X_{12}} F_{2}-\frac{X_{1} Z_{2}-X_{2} Z_{1}}{X_{12}} F_{4}, \\
& a_{42}=i \frac{2 Z_{1} Z_{2}}{\Gamma_{12}} F_{3}, \\
& a_{43}=-i \frac{Z_{1}+Z_{2}}{X_{12}} F_{2}-\frac{Z_{1}-Z_{2}}{X_{12}} F_{4}, \\
& a_{44}=F_{1}-i \frac{\gamma_{1} Z_{2}+\gamma_{2} Z_{1}}{\Gamma_{12}} F_{3} .
\end{aligned}
$$


The pairs $\left(r_{1}, r_{1}\right),\left(\gamma_{1}, \gamma_{2}\right),\left(X_{1}, X_{2}\right),\left(Z_{2}, Z_{2}\right)$ etc. are complex conjugates. Sum of the conjugates is real and difference is imaginary. Further multiplication of complex conjugates is real. Thus each element of (28) is real.

\section{Dispersion equation}

\subsection{Solid layers}

Let us consider $N$ homogeneous VTI layers numbering 1 to $N$ from top to bottom, the Nth layer being the homogeneous half space. The $m$ th layer is bounded by $(m-1)$ th interface at the top and $m$ th interface at the bottom. We use suffix $m$ to show the values in the $m$ th layer and superscript $m$ in bracket to show the values at the interface $m$.

The constants $\left(B_{1}\right)_{N}$ and $\left(B_{2}\right)_{N}$ are zero since displacement is zero at infinite depth $z=-\infty$. From (12), we have at the top of $\mathrm{Nth}$ layer

or

$$
\left[Y_{N}(0)\right]^{(N-1)}=\left[E_{N}(0)\right]\left[A_{1}, A_{1}, A_{2}, A_{2},\right]_{N}^{T}
$$

$$
\left[A_{1}, A_{1}, A_{2}, A_{2},\right]_{N}^{T}=\left[E_{N}(0)\right]^{-1}\left[Y_{N}(0)\right]^{(N-1)}
$$

Following Haskell [1953], we use continuity of displacement-stress matrix at interfaces and use the relation (17) to obtain

$$
\begin{gathered}
{\left[A_{1}, A_{1}, A_{2}, A_{2,}\right]_{N}^{T}=\left[E_{N}(0)\right]^{-1}\left[a_{N-1}\right] \ldots \ldots \ldots\left[a_{2}\right]\left[a_{1}\right]\left[Y_{1}(0)\right]} \\
=\left[E_{N}(0)\right]^{-1}[M]\left[Y_{1}(0)\right]=[J]\left[Y_{1}(0)\right]
\end{gathered}
$$

where the displacement-stress matrix $\left[Y_{1}(0)\right]$ at the free surface is

$$
\left[Y_{1}(0)\right]=\left[U_{0},-i W_{0}, 0,0\right]^{T}
$$

with $U_{0}=U(0)$ and $W_{0}=W(0)$. In (30)

$$
[M]=\left[a_{N-1}\right] \ldots \ldots \ldots\left[a_{2}\right]\left[a_{1}\right]
$$

and

$$
[J]=\left[E_{N}(0)\right]^{-1}[M] .
$$

Equating the rows in (30) and eliminating $U_{0}, W_{0}$, $A_{1 N}$ and $A_{2 N}$, we get the dispersion equation as

$$
\frac{J_{11}-J_{21}}{J_{31}-J_{41}}=\frac{J_{12}-J_{22}}{J_{32}-J_{42}}
$$

Multiplying both sides of (32) by

$$
\left[\begin{array}{cccc}
1 & -1 & 0 & 0 \\
0 & 0 & 1 & -1
\end{array}\right]
$$

and using (19) we get

$\left[\begin{array}{llll}J_{11}-J_{21} & J_{12}-J_{22} & J_{13}-J_{23} & J_{14}-J_{24} \\ J_{31}-J_{41} & J_{32}-J_{42} & J_{33}-J_{43} & J_{34}-J_{44}\end{array}\right]=\left[\begin{array}{cccc}-\frac{X_{2}}{X_{12}} & -\frac{Z_{2}}{\Gamma_{12}} & \frac{1}{X_{12}} & \frac{\gamma_{2}}{\Gamma_{12}} \\ \frac{X_{1}}{X_{12}} & \frac{Z_{1}}{\Gamma_{12}} & -\frac{1}{X_{12}} & -\frac{\gamma_{1}}{\Gamma_{12}}\end{array}\right]_{N}[M]$

The dispersion equation (33) may be written as

$$
\left(J_{11}-J_{21}\right)\left(J_{32}-J_{42}\right)-\left(J_{12}-J_{22}\right)\left(J_{31}-J_{41}\right)=0
$$

Equation (30) also gives surface ellipticity $\varepsilon$ as

$$
\varepsilon=\frac{U_{0}}{-i W_{0}}=-\frac{J_{12}-J_{22}}{J_{11}-J_{21}}
$$

\subsection{Liquid layer at the top}

Let us consider a liquid layer of density $\rho_{0}$, P-wave velocity $\alpha_{0}$ and thickness $H_{0}$ over the above $N$-layered VTI half space. The vertical components of displacement and stress in the liquid layer are respectively

$$
\begin{gathered}
W(z)=k r_{0} C_{0} \cos \left(k r_{0} z\right) \\
p_{z z}(z)=-\rho \omega^{2} C_{0} \sin \left(k r_{0} z\right)
\end{gathered}
$$

where $r_{0}=\sqrt{c^{2} / \alpha_{0}^{2}-1}$ [Ewing et al. 1957]. Thus in equation (30) instead of displacement-stress matrix $\left[Y_{1}(0)\right]$ on free surface, we use the displacement-stress matrix at the bottom $\left(z=-H_{0}\right)$ of the liquid layer as

$$
Y_{1}\left(-H_{0}\right)=\left[U_{0},-i k r_{0} C_{0} \cos \left(k r_{0} H_{0}\right), i C_{0}\left(\omega^{2} \rho / k\right) \sin \left(k r_{0} H_{0}\right), 0\right]^{T}
$$

Here $U_{0}$ is the horizontal displacement component in the top solid layer which is just below the liquid layer. As in section 4.1, equating the rows in equation (30) and eliminating $U_{0}, C_{0}, A_{1 \mathrm{~N}}$ and $A_{2 \mathrm{~N}}$ we get the dispersion equation as

$$
\frac{J_{12}-J_{22}}{J_{11}-J_{21}}-\frac{J_{32}-J_{42}}{J_{31}-J_{41}}-\chi\left[\frac{J_{13}-J_{23}}{J_{11}-J_{21}}-\frac{J_{33}-J_{43}}{J_{31}-J_{41}}\right]=0
$$

where

$$
\chi=\frac{\omega^{2} \rho}{k^{2} r_{0}} \tan \left(k r_{0} H_{0}\right)=\frac{\rho c^{2}}{r_{0}} \tan \left(k r_{0} H_{0}\right)
$$

Similar dispersion in isotropic layered half space with same $\chi$ was obtained by Harkrider (1964). The dispersion equation (37) can be written as

$$
\begin{gathered}
\left(J_{11}-J_{21}\right)\left(J_{32}-J_{42}\right)-\left(J_{12}-J_{22}\right)\left(J_{31}-J_{41}\right) \\
-\chi\left[\left(J_{11}-J_{21}\right)\left(J_{33}-J_{43}\right)-\left(J_{13}-J_{23}\right)\left(J_{31}-J_{41}\right)\right]=0
\end{gathered}
$$

If liquid layer is absent $H_{0}=0$ and so $\chi=0$; thus equation (39) has the same form as (35). As in section 4.1, the surface ellipticity at the top of solid layers is given by

$$
\varepsilon=\frac{U_{0}}{-i W_{0}}=-\frac{J_{12}-J_{22}}{J_{11}-J_{21}}+\chi \frac{J_{13}-J_{23}}{J_{11}-J_{21}}
$$




\section{Delta matrix}

We shall now obtain delta (compound) matrix [Thrower 1965, Harkrider 1970] of the layer matrix derived in section 3. We shall use a bar to denote a delta matrix e.g. delta matrix of $[a]$ is $[\bar{a}]$.

\subsection{Case I: $S_{3}^{2} \geq 0$}

Denoting

$C h_{j}=\cosh Q_{j}$ and $S h_{j}=\left(\sinh Q_{j}\right) / r_{j}$

the elements of the delta matrix $[\bar{a}]$ obtained from the matrix (25) are as follows.

$$
\begin{gathered}
\bar{a}_{11}=\left\{-X_{1}\left(1+g_{1}\right) g_{2}-X_{2}\left(1+g_{2}\right) g_{1}+C h_{1} C h_{2}\left[X_{1} g_{1}\left(1+g_{2}\right)+X_{2} g_{2}\left(1+g_{1}\right)\right]\right. \\
\left.-S h_{1} S h_{2}\left[X_{1} g_{2}\left(1+g_{2}\right) r_{2}^{2}+X_{2} g_{1}\left(1+g_{1}\right) r_{1}^{2}\right]\right\} /\left(X_{12} g_{12}\right) \\
\bar{a}_{12}=\left\{g_{2} r_{2}^{2} C h_{1} S h_{2}-g_{1} r_{1}^{2} C h_{2} S h_{1}\right\} / X_{12} \\
\bar{a}_{13}=\left\{g_{1} g_{2}\left(X_{1}+X_{2}\right)\left(1-C h_{1} C h_{2}\right)+S h_{1} S h_{2}\left[X_{1} g_{2}^{2} r_{2}^{2}+X_{2} g_{1}^{2} r_{1}^{2}\right]\right\} /\left(c_{44} g_{12} X_{12}\right) \\
\bar{a}_{14}=\left\{-\left(g_{1}+g_{2}+2 g_{1} g_{2}\right)\left(1-C h_{1} C h_{2}\right)-S h_{1} S h_{2}\left[\left(1+g_{2}\right) g_{2} r_{2}^{2}+\left(1+g_{1}\right) g_{1} r_{1}^{2}\right\} /\left(X_{12} g_{12}\right)\right. \\
=\bar{a}_{13} \\
\bar{a}_{15}=\left\{C h_{1} S h_{2} g_{1}-C h_{2} S h_{1} g_{2}\right\} /\left(C_{44} g_{12}\right)
\end{gathered}
$$

$\bar{a}_{16}=\left\{-g_{1} g_{2}\left(C h_{1}-C h_{2}\right)^{2}+\left(g_{2} S h_{1}-g_{1} S h_{2}\right)\left(g_{1} r_{1}^{2} S h_{1}-g_{2} r_{2}^{2} S h_{2}\right)\right\} /\left(c_{44} g_{12} X_{12}\right)$ $\bar{a}_{21}=\left\{\left(1+g_{1}\right) X_{2} C h_{1} S h_{2}-\left(1+g_{2}\right) X_{1} C h_{2} S h_{1}\right\} / g_{12}, \quad \bar{a}_{22}=C h_{1} C h_{2}$

$\bar{a}_{23}=\left\{X_{1} g_{2} C h_{2} S h_{1}-X_{2} g_{1} C h_{1} S h_{2}\right\} /\left(c_{44} g_{12}\right)$,

$\bar{a}_{24}=\left\{\left(1+g_{1}\right) C h_{1} S h_{2}-\left(1+g_{2}\right) C h_{2} S h_{1}\right\} / g_{12}=\bar{a}_{23}$,

$$
\bar{a}_{25}=-X_{12} S h_{1} S h_{2} /\left(c_{44} g_{12}\right), \quad \bar{a}_{26}=\bar{a}_{15},
$$$$
\bar{a}_{31}=\left\{-\left(1+g_{1}\right)\left(1+g_{2}\right)\left(X_{1}+X_{2}\right)\left(1-C h_{1} C h_{2}\right)\right.
$$$$
-S h_{1} S h_{2}\left[\left(1+g_{1}\right)^{2} r_{1}^{2} X_{2}+\left(1+g_{2}\right)^{2} r_{2}^{2} X_{1}\right\} c_{44} /\left(X_{12} g_{12}\right)
$$

$\bar{a}_{32}=\left\{-\left(1+g_{1}\right) r_{1}^{2} C h_{2} S h_{1}+\left(1+g_{2}\right) r_{2}^{2} C h_{1} S h_{2}\right\} c_{44} / X_{12}$ $\bar{a}_{33}=\left\{X_{1} g_{1}\left(1+g_{2}\right)+X_{2} g_{2}\left(1+g_{1}\right)-C h_{1} C h_{2}\left[X_{1}\left(1+g_{1}\right) g_{2}+X_{2}\left(1+g_{2}\right) g_{1}\right.\right.$ $\left.+S h_{1} S h_{2}\left[X_{1} g_{2} r_{2}^{2}\left(1+g_{2}\right)+X_{2} g_{1} r_{1}^{2}\left(1+g_{1}\right)\right] /\left(X_{12} g_{12}\right)\right]$

$\bar{a}_{34}=\left\{-2\left(1-C h_{1} C h_{2}\right)\left(1+g_{1}\right)\left(1+g_{2}\right)\right\}$

$\left.-S h_{1} S h_{2}\left[\left(1+g_{1}\right)^{2} r_{1}^{2}+\left(1+g_{2}\right)^{2} r_{2}^{2}\right]\right\} c_{44} /\left(X_{12} g_{12}\right)=\bar{a}_{33}-1$ $\bar{a}_{35}=\bar{a}_{24}, \quad \bar{a}_{36}=\bar{a}_{14}$

$\bar{a}_{41}=\left\{X_{1} X_{2}\left[g_{1}\left(1+g_{2}\right)+g_{2}\left(1+g_{1}\right)\right]\left(1-C h_{1} C h_{2}\right)\right.$ $\left.+S h_{1} S h_{2}\left[X_{1}^{2} g_{2}\left(1+g_{2}\right) r_{2}^{2}+X_{2}^{2} g_{1}\left(1+g_{1}\right) r_{1}^{2}\right]\right\} /\left(X_{12} g_{12}\right)=\bar{a}_{31}$ $\bar{a}_{42}=\left\{g_{1} r_{1}^{2} X_{2} C h_{2} S h_{1}-g_{2} r_{2}^{2} X_{1} C h_{1} S h_{2}\right\} / X_{12}=\bar{a}_{32}$ $\bar{a}_{43}=\left\{-2 X_{1} X_{2} g_{1} g_{2}\left(1-C h_{1} C h_{2}\right)-S h_{1} S h_{2}\left[X_{1}^{2} g_{2}^{2} r_{2}^{2}+X_{2}^{2} g_{1}^{2} r_{1}^{2}\right]\right\} /\left(c_{44} g_{12} X_{12}\right)=\bar{a}_{34}$

$$
\begin{gathered}
\bar{a}_{44}=\bar{a}_{33}, \quad \bar{a}_{45}=\bar{a}_{23}, \quad \bar{a}_{46}=\bar{a}_{13}, \\
\bar{a}_{51}=\left\{X_{1} r_{2}^{2}\left(1+g_{2}\right) C h_{1} S h_{2}-X_{2} r_{1}^{2}\left(1+g_{1}\right) C h_{2} S h_{1}\right\} c_{44} / X_{12} \\
\bar{a}_{52}=\left\{-r_{1}^{2} r_{2}^{2} g_{12} S h_{1} S h_{2}\right\} c_{44} / X_{12}
\end{gathered}
$$

$\bar{a}_{53}=\bar{a}_{42}, \quad \bar{a}_{54}=\bar{a}_{32}, \quad \bar{a}_{55}=\bar{a}_{22}, \quad \bar{a}_{56}=\bar{a}_{12}$,

$\bar{a}_{61}=\left\{-2 X_{1} X_{2}\left(1+g_{1}\right)\left(1+g_{2}\right)\left(1-C h_{1} C h_{2}\right)\right.$

$\left.-S h_{1} S h_{2}\left[X_{1}^{2}\left(1+g_{2}\right)^{2} r_{2}^{2}+X_{2}^{2}\left(1+g_{1}\right)^{2} r_{1}^{2}\right]\right\} c_{44} /\left(X_{12} g_{12}\right)$,

$\bar{a}_{62}=\bar{a}_{51}, \quad \bar{a}_{63}=\bar{a}_{41}, \quad \bar{a}_{64}=\bar{a}_{31}, \bar{a}_{65}=\bar{a}_{21}, \bar{a}_{66}=\bar{a}_{11}$.

5.2 Case II: $S_{3}^{2}<0$

We have already defined $F_{\mathrm{n}}$ in equations (27) for $\mathrm{n}=1,2,3,4$. Let us further define

$F_{5}=F_{1} F_{4}+F_{2} F_{3}=\cosh Q^{\prime} \sinh Q^{\prime}, F_{6}=F_{1} F_{2}-F_{3} F_{4}=\cos Q^{\prime \prime} \sin Q^{\prime \prime}$

It may be noted that

$$
F_{2} F_{4}-F_{1} F_{3}=0 \text {. }
$$

Thus from (28), the elements of delta matrix $[\bar{a}]$ are written as

$$
\begin{gathered}
\bar{a}_{11}=F_{1}^{2}-\frac{Z_{1}+Z_{2}}{\Gamma_{12}} \frac{X_{1} \gamma_{2}+X_{2} \gamma_{1}}{X_{12}} F_{2}^{2}+\frac{X_{1}+X_{2}}{X_{12}} \frac{\gamma_{1} Z_{2}+\gamma_{2} Z_{1}}{\Gamma_{12}} F_{3}^{2}+\frac{Z_{1}-Z_{2}}{\Gamma_{12}} \frac{X_{1} \gamma_{2}-X_{2} \gamma_{1}}{X_{12}} F_{4}^{2} \\
\bar{a}_{12}=-\frac{\gamma_{1}-\gamma_{2}}{X_{12}} F_{5}-i \frac{\gamma_{1}+\gamma_{2}}{X_{12}} F_{6}
\end{gathered}
$$

$\bar{a}_{13}=\frac{\gamma_{1}+\gamma_{2}}{\Gamma_{12}} \frac{X_{1} \gamma_{2}+X_{2} \gamma_{1}}{X_{12}} F_{2}^{2}-\frac{X_{1}+X_{2}}{X_{12}} \frac{2 \gamma_{1} \gamma_{2}}{\Gamma_{12}} F_{3}^{2}-\frac{\gamma_{1}-\gamma_{2}}{\Gamma_{12}} \frac{X_{1} \gamma_{2}-X_{2} \gamma_{1}}{X_{12}} F_{4}^{2}$

$$
\bar{a}_{14}=-\frac{\gamma_{1}+\gamma_{2}}{X_{12}} \frac{Z_{1}+Z_{2}}{\Gamma_{12}} F_{2}^{2}+\frac{2\left(\gamma_{1} Z_{2}+\gamma_{2} Z_{1}\right)}{X_{12} \Gamma_{12}} F_{3}^{2}-\frac{\gamma_{1}-\gamma_{2}}{X_{12}} \frac{Z_{1}-Z_{2}}{\Gamma_{12}} F_{4}^{2}=\bar{a}_{13}
$$

$$
\bar{a}_{15}=\frac{\gamma_{1}-\gamma_{2}}{\Gamma_{12}} F_{5}-i \frac{\gamma_{1}+\gamma_{2}}{\Gamma_{12}} F_{6},
$$

$$
\bar{a}_{16}=-\frac{\left(\gamma_{1}+\gamma_{2}\right)^{2}}{X_{12} \Gamma_{12}} F_{2}^{2}+\frac{4 \gamma_{1} \gamma_{2}}{X_{12} \Gamma_{12}} F_{3}^{2}-\frac{\left(\gamma_{1}-\gamma_{2}\right)^{2}}{X_{12} \Gamma_{12}} F_{4}^{2},
$$

$$
\bar{a}_{21}=-\frac{X_{1} Z_{2}-X_{2} Z_{1}}{\Gamma_{12}} F_{5}-i \frac{X_{1} Z_{2}+X_{2} Z_{1}}{\Gamma_{12}} F_{6}
$$

$$
\bar{a}_{22}=F_{1}^{2}+F_{3}^{2},
$$

$$
\begin{gathered}
\bar{a}_{23}=\frac{X_{1} \gamma_{2}-X_{2} \gamma_{1}}{\Gamma_{12}} F_{5}+i \frac{X_{1} \gamma_{2}+X_{2} \gamma_{1}}{\Gamma_{12}} F_{6}, \\
\bar{a}_{24}=\frac{Z_{1}-Z_{2}}{\Gamma_{12}} F_{5}-i \frac{Z_{1}+Z_{2}}{\Gamma_{12}} F_{6}=\bar{a}_{23},
\end{gathered}
$$

$$
\bar{a}_{25}=-\frac{X_{1}-X_{2}}{\Gamma_{12}}\left(F_{2}^{2}+F_{4}^{2}\right), \quad \bar{a}_{26}=\bar{a}_{15},
$$

$\bar{a}_{31}=-\frac{Z_{1}+Z_{2}}{\Gamma_{12}} \frac{X_{1} Z_{2}+X_{2} Z_{1}}{X_{12}} F_{2}^{2}+\frac{2 Z_{1} Z_{2}\left(X_{1}+X_{2}\right)}{X_{12} \Gamma_{12}} F_{3}^{2}+\frac{Z_{1}-Z_{2}}{\Gamma_{12}} \frac{X_{1} Z_{2}-X_{2} Z_{1}}{X_{12}} F_{4}^{2}$,

$$
\bar{a}_{32}=-\frac{Z_{1}-Z_{2}}{X_{12}} F_{5}-i \frac{Z_{1}+Z_{2}}{X_{12}} F_{6},
$$

$\bar{a}_{33}=F_{1}^{2}+\frac{\gamma_{1}+\gamma_{2}}{\Gamma_{12}} \frac{X_{1} Z_{2}+X_{2} Z_{1}}{X_{12}} F_{2}^{2}-\frac{X_{1}+X_{2}}{X_{12}} \frac{\gamma_{1} Z_{2}+\gamma_{2} Z_{1}}{\Gamma_{12}} F_{3}^{2}-\frac{\gamma_{1}-\gamma_{2}}{\Gamma_{12}} \frac{X_{1} Z_{2}-X_{2} Z_{1}}{X_{12}} F_{4}^{2}$,

$$
\begin{gathered}
\bar{a}_{34}=-\frac{\left(Z_{1}+Z_{2}\right)^{2}}{X_{12} \Gamma_{12}} F_{2}^{2}+\frac{4 Z_{1} Z_{2}}{X_{12} \Gamma_{12}} F_{3}^{2}-\frac{\left(Z_{1}-Z_{2}\right)^{2}}{X_{12} \Gamma_{12}} F_{4}^{2}, \\
\bar{a}_{35}=\bar{a}_{24}, \quad \bar{a}_{36}=\bar{a}_{14},
\end{gathered}
$$

$\bar{a}_{41}=\frac{X_{1} \gamma_{2}+X_{2} \gamma_{1}}{X_{12}} \frac{X_{1} Z_{2}+X_{2} Z_{1}}{\Gamma_{12}} F_{2}^{2}-\frac{2 X_{1} X_{2}}{X_{12}} \frac{\gamma_{1} Z_{2}+\gamma_{2} Z_{1}}{\Gamma_{12}} F_{3}^{2}+\frac{X_{1} \gamma_{2}-X_{2} \gamma_{1}}{X_{12}} \frac{X_{1} Z_{2}-X_{2} Z_{1}}{\Gamma_{12}} F_{4}^{2}=\bar{a}_{31}$

$$
\bar{a}_{42}=-\frac{X_{1} \gamma_{2}-X_{2} \gamma_{1}}{X_{12}} F_{5}+i \frac{X_{1} \gamma_{2}+X_{2} \gamma_{1}}{X_{12}} F_{6}=\bar{a}_{32},
$$

$\bar{a}_{43}=-\frac{\left(X_{1} \gamma_{2}+X_{2} \gamma_{1}\right)^{2}}{X_{12} \Gamma_{12}} F_{2}^{2}+4 \frac{\gamma_{1} \gamma_{2}}{\Gamma_{12}} \frac{X_{1} X_{2}}{X_{12}} F_{3}^{2}-\frac{\left(X_{1} \gamma_{2}-X_{2} \gamma_{1}\right)^{2}}{X_{12} \Gamma_{12}} F_{4}^{2}=\bar{a}_{34}$, 


$$
\begin{gathered}
\bar{a}_{44}=\bar{a}_{33}, \quad \bar{a}_{45}=\bar{a}_{23}, \quad \bar{a}_{46}=\bar{a}_{13}, \\
\bar{a}_{51}=\frac{X_{1} Z_{2}-X_{2} Z_{1}}{X_{12}} F_{5}-i \frac{X_{1} Z_{2}+X_{2} Z_{1}}{X_{12}} F_{6}, \bar{a}_{52}=-\frac{\Gamma_{12}}{X_{12}}\left(F_{4}^{2}+F_{2}^{2}\right), \\
\bar{a}_{53}=\bar{a}_{42}, \quad \bar{a}_{54}=\bar{a}_{32}, \quad \bar{a}_{55}=\bar{a}_{22}, \quad \bar{a}_{56}=\bar{a}_{12}, \\
\bar{a}_{61}=-\frac{\left(X_{1} Z_{2}+X_{2} Z_{1}\right)^{2}}{X_{12} \Gamma_{12}} F_{2}^{2}+4 \frac{X_{1} X_{2}}{X_{12}} \frac{Z_{1} Z_{2}}{\Gamma_{12}} F_{3}^{2}-\frac{\left(X_{1} Z_{2}-X_{2} Z_{1}\right)^{2}}{X_{12} \Gamma_{12}} F_{4}^{2}, \\
\bar{a}_{62}=\bar{a}_{51}, \quad \bar{a}_{63}=\bar{a}_{41}, \bar{a}_{64}=\bar{a}_{31}, \bar{a}_{65}=\bar{a}_{21}, \bar{a}_{66}=\bar{a}_{11} .
\end{gathered}
$$

Based on the arguments given below Equation (28), each term in each of the above matrix element is real.

\section{Dispersion equation in terms of delta matrix}

We write the equation (34) as

$$
[K]=[P][M]
$$

where

$$
[K]=\left[\begin{array}{llll}
J_{11}-J_{21} & J_{12}-J_{22} & J_{13}-J_{23} & J_{14}-J_{24} \\
J_{31}-J_{41} & J_{32}-J_{42} & J_{33}-J_{43} & J_{34}-J_{44}
\end{array}\right],
$$

and

$$
[P]=\left[\begin{array}{cccc}
-\frac{X_{2}}{X_{12}} & -\frac{Z_{2}}{\Gamma_{12}} & \frac{1}{X_{12}} & \frac{\gamma_{2}}{\Gamma_{12}} \\
\frac{X_{1}}{X_{12}} & \frac{Z_{1}}{\Gamma_{12}} & -\frac{1}{X_{12}} & -\frac{\gamma_{1}}{\Gamma_{12}}
\end{array}\right]_{N}
$$

Using the properties of delta matrix, from (43) we get

$$
[\bar{K}]=[\bar{P}][\bar{M}]
$$

where $[\bar{K}]$ and $[\bar{P}]$ are $(1 \times 6)$ matrices with elements

$$
\begin{aligned}
& \bar{K}_{11}=\left(J_{11}-J_{21}\right)\left(J_{32}-J_{42}\right)-\left(J_{12}-J_{22}\right)\left(J_{31}-J_{41}\right), \\
& \left.\bar{K}_{12}=\left(J_{11}-J_{21}\right)\left(J_{33}-J_{43}\right)-\left(J_{13}-J_{22}\right) J_{31}-J_{41}\right), \text { etc } . \\
& {[\bar{P}]=\left[\frac{X_{1} Z_{2}-X_{2} Z_{1}}{X_{12} \Gamma_{12}}, \frac{1}{X_{12}},-\frac{X_{1} \gamma_{2}-X_{2} \gamma_{1}}{X_{12} \Gamma_{12}},-\frac{Z_{1}-Z_{2}}{X_{12} \Gamma_{12}}, \frac{1}{\Gamma_{12}},-\frac{\gamma_{1}-\gamma_{2}}{X_{12} \Gamma_{12}}\right]_{N},}
\end{aligned}
$$

and

$$
[\bar{M}]=\left[\bar{a}_{N-1}\right]\left[\bar{a}_{N-2}\right] \ldots \ldots \ldots\left[\bar{a}_{3}\right]\left[\bar{a}_{2}\right]\left[\bar{a}_{1}\right] .
$$

The dispersion equation (39) is written as

$$
\bar{K}_{11}-\chi \bar{K}_{12}=0
$$

where $\chi=0$ when top liquid layer is absent i.e. when $H_{0}$ $=0$. Using (44), the above dispersion equation can be written as

$$
[\bar{P}]\left[\bar{a}_{N-1}\right]\left[\bar{a}_{N-2}\right] \ldots \ldots \ldots\left[\bar{a}_{3}\right]\left[\bar{a}_{2}\right]\left[\hat{a}_{1}\right]=0
$$

where $\left[\hat{a}_{1}\right]$ is a $(6 \times 1)$ matrix given by

$\left[\hat{a}_{1}\right]=\left[\bar{a}_{11}-\chi \bar{a}_{12}, \bar{a}_{21}-\chi \bar{a}_{22}, \bar{a}_{31}-\chi \bar{a}_{32}, \bar{a}_{41}-\chi \bar{a}_{42}, \bar{a}_{51}-\chi \bar{a}_{52}, \bar{a}_{61}-\chi \bar{a}_{62}\right]_{1}^{T}$

If $r_{j}$ is complex in the Nth layer, each term of $[\bar{P}]$ has a factor $i$, which gets eliminated in equation (45). $\left[\bar{a}_{m}\right]$ has already been seen as real $(m=1,2, \ldots$, $\mathrm{N}-1$ ). Thus the dispersion equation (45) contains all real terms.

Relationships among the elements of delta matrix $[\bar{a}]$ and $\bar{P}_{13}=\bar{P}_{14}$ show that $\bar{K}_{13}=\bar{K}_{14}$. Following Harkrider [1964, Equation 74], we write surface ellipticity $\varepsilon$ from equation (36) as

$$
\varepsilon=-\bar{K}_{13} / \bar{K}_{12}
$$

which is real even if $r_{j}$ is complex. The above expression for $\varepsilon$ is also obtained from equation (40) at the top of solid layers below the liquid layer.

\section{Dispersion equation in terms of reduced delta matrix}

The relationship among the elements of $\left[\bar{a}_{\mathrm{m}}\right]$ have been indicated in equations (41) and (42); further $\bar{P}_{13}=\bar{P}_{14}$. Thus the dispersion equation (45) can be written as

$$
[\overline{\bar{P}}]\left[\overline{\bar{a}}_{N-1}\right]\left[\overline{\bar{a}}_{N-2}\right] \ldots \ldots \ldots\left[\overline{\bar{a}}_{3}\right]\left[\overline{\bar{a}}_{2}\right]\left[\check{a}_{1}\right]=0
$$

where the reduced delta matrix $\left[\overline{\bar{a}}_{m}\right]$ of $m$ th layer is given by

$$
\left[\overline{\bar{a}}_{m}\right]=\left[\begin{array}{ccccc}
\bar{a}_{11} & \bar{a}_{12} & 2 \bar{a}_{13} & \bar{a}_{15} & \bar{a}_{16} \\
\bar{a}_{21} & \bar{a}_{22} & 2 \bar{a}_{23} & \bar{a}_{25} & \bar{a}_{26} \\
\bar{a}_{31} & \bar{a}_{32} & \bar{a}_{33}+\bar{a}_{34} & \bar{a}_{35} & \bar{a}_{36} \\
\bar{a}_{51} & \bar{a}_{52} & 2 \bar{a}_{53} & \bar{a}_{55} & \bar{a}_{56} \\
\bar{a}_{61} & \bar{a}_{62} & 2 \bar{a}_{63} & \bar{a}_{65} & \bar{a}_{66}
\end{array}\right]_{m}
$$

In equation (47)

$$
[\overline{\bar{P}}]=\left[\bar{P}_{11}, \bar{P}_{12}, 2 \bar{P}_{13}, \bar{P}_{15}, \bar{P}_{16}\right]
$$

and

$$
\left[\breve{a}_{1}\right]=\left[\overline{\bar{a}}_{11}-\chi \overline{\bar{a}}_{12}, \overline{\bar{a}}_{21}-\chi \overline{\bar{a}}_{22}, \overline{\bar{a}}_{31}-\chi \overline{\bar{a}}_{32}, \overline{\bar{a}}_{41}-\chi \overline{\bar{a}}_{42}, \overline{\bar{a}}_{51}-\chi \overline{\bar{a}}_{52}\right]_{1}^{T}
$$

In terms of reduced layer matrix surface ellipticity $\varepsilon$ in equation (46) can be written as

$$
\varepsilon=-0.5 \bar{K}_{3} / \bar{K}_{2}
$$

where

$$
\overline{\bar{K}}_{j}=[\overline{\bar{P}}]\left[\overline{\bar{a}}_{N-1}\right]\left[\overline{\bar{a}}_{N-2}\right] \ldots \ldots \ldots\left[\overline{\bar{a}}_{3}\right]\left[\overline{\bar{a}}_{2}\right]\left[\tilde{a}_{1(j)}\right]
$$

with $\mathrm{j}=2,3$ and $\left[\tilde{a}_{1(\mathrm{j})}\right]$ is the $\mathrm{jth}$ column of $\left[\overline{\bar{a}}_{1}\right]$.

\section{Computational results and discussions}

Based on dispersion equation (47), a FORTRAN code in real domain is prepared to evaluate phase and 
group velocities; surface ellipticity of Rayleigh waves is obtained using equation (48). Considering dispersion equation (47) as $f(k, c)=0$, group velocity $(\mathrm{U})$ is obtained using

$$
U=c-k \frac{(\partial f / \partial k)_{c}}{(\partial f / \partial c)_{k}}
$$

where the above partial derivatives in the numerator and denominator are obtained analytically from $f$. The code may be shared on request. Using this code, dispersion curves are drawn here in Figures 2, 3 and 5 for a few existing oceanic and continental VTI models. Through generalized Reflection-Transmission method, Bhattacharya and Arora [1997] obtained dispersion equation with complex terms in a layered VTI spherical earth using a flattening transformation. This method to get dispersion curves in a layered VTI half space works in a complex domain and gives the same results as from the present code.

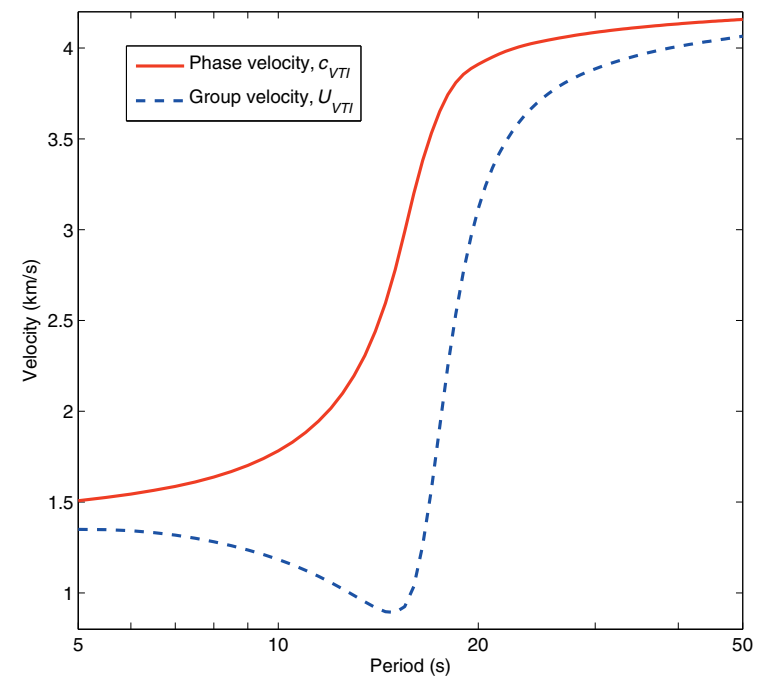

Figure 2. Phase and group velocities of fundamental mode Rayleigh waves for the isotropic crust over anisotropic upper mantle of the Pacific region of age 110+ Myr (Table 1a).

\subsection{Oceanic structure}

Let us consider a crust and upper mantle structure of the Pacific region of age 110+ Myr [Nishimura and Forsyth, 1989]. We consider 5 layers for isotropic crust over 5 layers of VTI subcrust (Table 1a). Figure 2 shows the phase $\left(\mathrm{c}_{\mathrm{VTI}}\right)$ and group $\left(\mathrm{U}_{\mathrm{VTI}}\right)$ velocities of fundamental mode Rayleigh waves. During computation, complex $r_{j}$ appears in subcrustal layers for periods up to $18 \mathrm{~s}$. The Table $1 \mathrm{~b}$ gives the numerical values of $\mathrm{c}_{\mathrm{VTI}}$, $\mathrm{U}_{\mathrm{VTI}}$ and $\varepsilon_{\mathrm{VTI}}$, which can be used to test other codes.

\subsection{Continental structure}

As a continental structure, let us consider the VTI model obtained by Huang et al. [2010] for SW China using Love and Rayleigh waves up to the period $40 \mathrm{~s}$.

\begin{tabular}{lcccccc}
\hline Layer No. & $\begin{array}{c}\text { Thickness } \\
(\mathbf{k m})\end{array}$ & $\begin{array}{c}\alpha_{\mathrm{H}} \\
(\mathbf{k m} / \mathbf{s})\end{array}$ & $\begin{array}{c}\beta_{\mathrm{v}} \\
(\mathbf{k m} / \mathbf{s})\end{array}$ & $\phi$ & $\eta$ & $\begin{array}{c}\rho \\
\mathbf{g m} / \mathbf{c m}^{3}\end{array}$ \\
\hline 1 & 6.1 & 1.5000 & 0.0000 & 1.0000 & 1.0000 & 1.03 \\
2 & 0.3 & 2.0000 & 0.5000 & 1.0000 & 1.0000 & 1.50 \\
3 & 1.0 & 5.6000 & 3.3000 & 1.0000 & 1.0000 & 2.60 \\
4 & 2.0 & 6.4000 & 3.7000 & 1.0000 & 1.0000 & 2.80 \\
5 & 3.0 & 7.2000 & 4.1000 & 1.0000 & 1.0000 & 3.00 \\
6 & 2.6 & 8.1780 & 4.6357 & 0.9868 & 1.0000 & 3.3995 \\
7 & 5.0 & 8.1747 & 4.6232 & 0.9786 & 1.0024 & 3.3924 \\
8 & 10.0 & 8.1699 & 4.6051 & 0.9666 & 1.0094 & 3.3819 \\
9 & 10.0 & 8.1677 & 4.5947 & 0.9620 & 1.0176 & 3.3669 \\
10 & $\infty$ & 8.1618 & 4.6222 & 0.9712 & 1.0169 & 3.3530 \\
\hline
\end{tabular}

Table 1a. Structure of the Pacific region with age 110+ Myr [based on Nishimura and Forsyth, 1989].

\begin{tabular}{lccc}
\hline Period $(\mathbf{s})$ & $c_{\mathrm{VTI}}(\mathrm{km} / \mathrm{s})$ & $U_{\mathrm{VTI}}(\mathrm{km} / \mathrm{s})$ & $\varepsilon_{\mathrm{VTI}}$ \\
\hline 5.000 & 1.5078 & 1.3497 & 0.9109 \\
10.000 & 1.7819 & 1.1842 & 0.1457 \\
15.000 & 2.7796 & 0.8923 & -0.2401 \\
20.000 & 3.9105 & 3.1159 & -0.6831 \\
25.000 & 4.0378 & 3.7239 & -0.7429 \\
30.000 & 4.0863 & 3.8858 & -0.7505 \\
35.000 & 4.1143 & 3.9625 & -0.7483 \\
40.000 & 4.1333 & 4.0092 & -0.7438 \\
50.000 & 4.1578 & 4.0654 & -0.7346 \\
\hline
\end{tabular}

Table 1b. Numerical values of phase velocity $\left(c_{\mathrm{VTI}}\right)$, group velocity $\left(U_{\mathrm{VTI}}\right)$ and surface ellipticity $\left(\varepsilon_{\mathrm{VTI}}\right)$ of fundamental mode of Rayleigh wave at a few sample periods for the oceanic VTI model in Table 1a.

\begin{tabular}{lccccccc}
\hline $\begin{array}{l}\text { Layer } \\
\text { No. }\end{array}$ & $\begin{array}{c}\text { Thickness } \\
(\mathbf{k m})\end{array}$ & $\begin{array}{c}\alpha_{\mathrm{H}} \\
(\mathbf{k m} / \mathbf{s})\end{array}$ & $\begin{array}{c}\beta_{\mathrm{H}} \\
(\mathbf{k m} / \mathbf{s})\end{array}$ & $\begin{array}{c}\beta_{\mathrm{V}} \\
(\mathbf{k m} / \mathbf{s})\end{array}$ & $\phi$ & $\eta$ & $\begin{array}{c}\rho \\
\mathbf{g m} / \mathbf{c m}^{3}\end{array}$ \\
\hline 1 & 16.0 & 5.63 & 3.25 & 3.20 & 0.9695 & 1.02 & 2.5 \\
2 & 16.0 & 6.15 & 3.55 & 3.34 & 0.8852 & 1.02 & 2.7 \\
3 & 17.0 & 6.70 & 3.87 & 3.63 & 0.8798 & 1.02 & 3.0 \\
4 & $\infty$ & 7.70 & 4.39 & 4.28 & 0.9505 & 1.01 & 3.3 \\
\hline
\end{tabular}

Table 2a. Example of a VTI continental structure [based on Huang et al. 2010].

\begin{tabular}{lccc}
\hline Period $(\mathbf{s})$ & $c_{\text {VTI }}(\mathrm{km} / \mathrm{s})$ & $U_{\text {VTI }}(\mathrm{km} / \mathbf{s})$ & $\varepsilon_{\text {VTI }}$ \\
\hline 5.000 & 2.9389 & 2.9264 & -0.6765 \\
10.000 & 2.9716 & 2.8784 & -0.6719 \\
15.000 & 3.0323 & 2.8244 & -0.6691 \\
20.000 & 3.1229 & 2.7448 & -0.6664 \\
25.000 & 3.2457 & 2.6857 & -0.6650 \\
30.000 & 3.3844 & 2.7254 & -0.6715 \\
35.000 & 3.5085 & 2.8842 & -0.6914 \\
40.000 & 3.5996 & 3.0878 & -0.7219 \\
50.000 & 3.7011 & 3.3980 & -0.7870 \\
\hline
\end{tabular}

Table 2b. Numerical values of phase velocity $\left(c_{\mathrm{VTI}}\right)$, group velocity $\left(U_{\mathrm{VTI}}\right)$ and surface ellipticity $\left(\varepsilon_{\mathrm{VTI}}\right)$ of fundamental mode of Rayleigh wave at a few sample periods for the continental VTI model in Table 2a.

For the model, they evaluated only $\beta_{\mathrm{H}}$ and $\beta_{\mathrm{V}}$ considering $\xi=\phi=\eta=1$. We have adopted this model and 

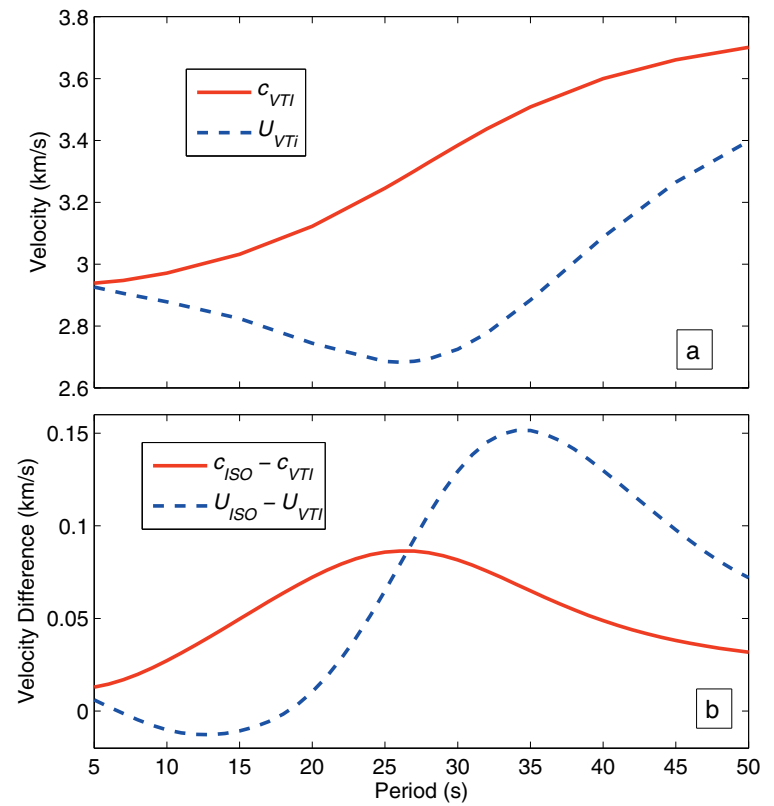

Figure 3. (a) Phase velocity $\left(c_{\mathrm{VTI}}\right)$ and group velocity $\left(U_{\mathrm{VTI}}\right)$ of fundamental mode Rayleigh waves for the continental VTI structure in Table 2a. (b) $c_{\text {ISO }}$ and $U_{\text {ISO }}$ are respectively phase and group velocities of fundamental mode Rayleigh waves for an isotropic structure with $\phi=\eta=1$ in all the layers in Table $2 \mathrm{a}$. The differences $\left(c_{\mathrm{ISO}}-c_{\mathrm{VTI}}\right)$ and $\left(U_{\mathrm{ISO}}-U_{\mathrm{VTI}}\right)$ are shown.

considered $\alpha_{\mathrm{H}}=1.732 \beta_{\mathrm{H}}$ and $\alpha_{\mathrm{V}}=1.732 \beta_{\mathrm{V}}$; thus $\phi=\left[\alpha_{\mathrm{V}} / \alpha_{\mathrm{H}}\right]^{2} ; \beta_{\mathrm{H}}$ or $\xi$ has no effect on Rayleigh waves. A small value of $\eta$ has been considered in the adopted model (Table 2a). For this model Figure 3a shows the phase velocities $\left(c_{\mathrm{VTI}}\right)$ and group velocities $\left(U_{\mathrm{VTI}}\right)$ of fundamental mode Rayleigh waves. During computation, complex $r_{1}$ and $r_{2}$ appear at least in one of the lower layers up to period $39 \mathrm{~s}$. An isotropic model is considered with $\phi=\eta=1$ in Table $2 \mathrm{a}$ and the corresponding phase velocities $\left(c_{\text {ISO }}\right)$ and group velocities $\left(U_{\text {ISO }}\right)$ are obtained. The difference $\left(c_{\text {ISO }}-c_{\mathrm{VTI}}\right)$ is as large as $0.086 \mathrm{~s}$ around the period $26 \mathrm{~s}$; further, the difference $\left(U_{\text {ISO }}-U_{\mathrm{VTI}}\right)$ is as large as 0.15 around the period $35 \mathrm{~s}$ (Figure $3 \mathrm{~b}$ ). Surface ellipticity $(\varepsilon)$ is shown in Figure 4 . The table $2 \mathrm{~b}$ gives the numerical values of $c_{\mathrm{VTI}}, U_{\mathrm{VTI}}$ and $\varepsilon_{\mathrm{VTI}}$.

As indicated in the Introduction, there are a few studies where observed surface wave velocities are initially inverted to get an isotropic model for which theoretical dispersion curves for Love and Rayleigh waves are plotted along with the observed velocity data. In a region of positive $S$-wave anisotropy $\left(\beta_{\mathrm{H}}>\beta_{\mathrm{V}}\right)$, these theoretical velocities for Love waves are lesser than the observed data, while those for Rayleigh waves are more than the data on an average. The fit of theoretical curves to the observed data is improved by considering S-wave velocity as $\beta_{\mathrm{H}}$ with $\xi=1$ for Love waves and Swave velocity as $\beta_{\mathrm{V}}$ with $\phi=\eta=1$ for Rayleigh waves with $\beta_{\mathrm{H}}>\beta_{\mathrm{V}}$. In such a case, we can use the code of a

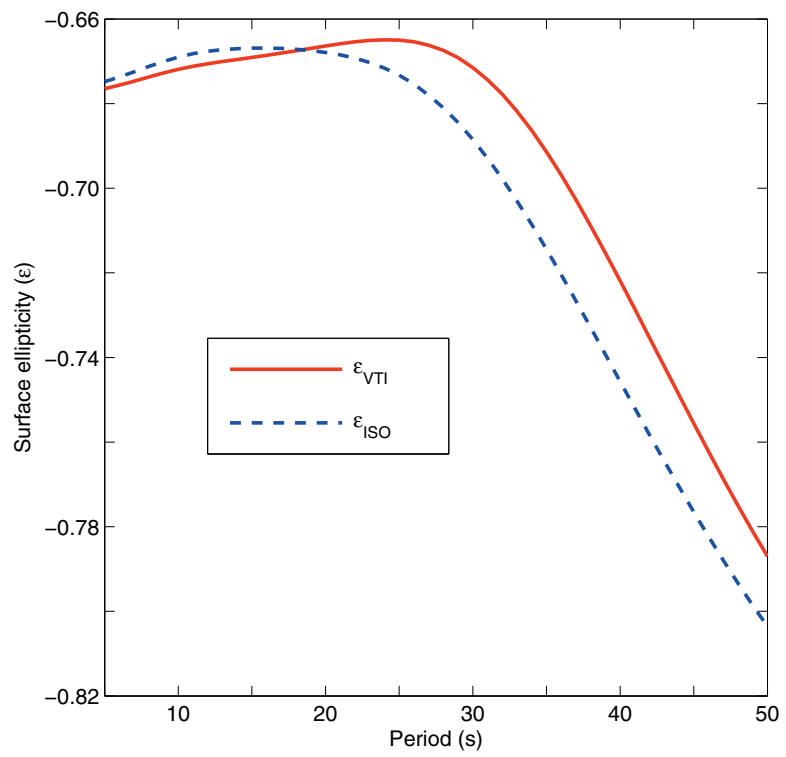

Figure 4. Surface ellipticity $\left(\varepsilon_{\mathrm{VTI}}\right)$ of fundamental mode Rayleigh waves for the continental VTI structure in Table 2a. Surface ellipticity $\left(\varepsilon_{\text {ISO }}\right)$ is also shown for an isotropic structure with $\phi=\eta=1$ in all the layers in Table $2 \mathrm{a}$.
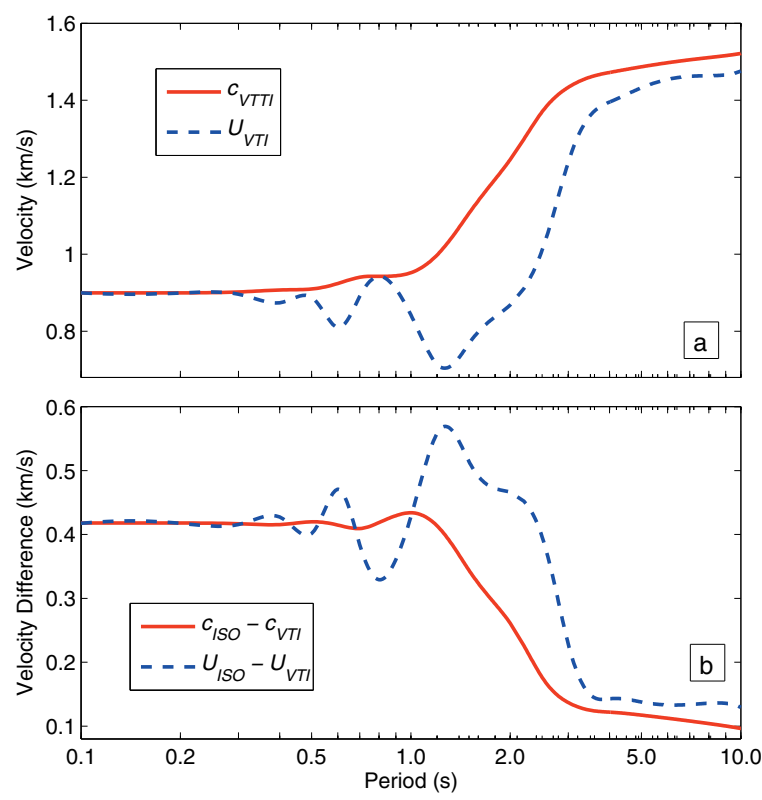

Figure 5. (a) Phase velocity $\left(c_{\mathrm{VTI}}\right)$ and group velocity $\left(U_{\mathrm{VTI}}\right)$ of fundamental mode Rayleigh waves for the subsurface VTI structure in Table 3. (b) $c_{\text {ISO }}$ and $U_{\text {ISO }}$ are respectively phase and group velocities of fundamental mode Rayleigh waves for the isotropic structure with $\phi=\eta=1$ in all the layers in Table 3 . The differences $\left(c_{\mathrm{ISO}}-c_{\mathrm{VTI}}\right)$ and $\left(U_{\mathrm{ISO}}-U_{\mathrm{VTI}}\right)$ are shown.

layered isotropic half space. However, Figure $3 \mathrm{~b}$ shows that consideration of $\phi \neq 1$ and $\eta \neq 1$ significantly changes the Rayleigh wave velocities. Thus reducing Rayleigh wave theoretical velocities only by $\beta_{\mathrm{V}}$ may give lower $\beta_{\mathrm{V}}$ than actual and this overestimates $S$-wave anisotropy $\xi$. This was also explained by Bhattacharya [2015] through sensitivity of Love and Rayleigh wave velocities to VTI medium parameters. A similar erro- 
neous estimate of $\xi$ may be obtained in a region with negative $S$-wave anisotropy $\left(\beta_{\mathrm{H}}<\beta_{\mathrm{V}}\right)$ for considering $\phi=\eta=1$.

\subsection{Subsurface structure}

Surface waves have been used for near surface studies [Socco et al. 2010, Dal Moro 2014]. Consideration of VTI gives better subsurface structure in exploration seismology. In a VTI subsurface, Levin [1979] considered reflections from interfaces. Thomsen [1986] derived relations between NMO (normal move out) velocities and anisotropic parameters. These studies encouraged to obtain subsurface structure through anisotropic travel time inversion and NMO velocity analysis [Tsvankin and Thomsen 1995, Alkalifah and Tsvankin 1995]. In such studies the five stiff elastic coefficients of VTI medium are replaced by $\alpha_{0}, \beta_{0}$ and the Thomsen parameters $\gamma, \varepsilon_{T}$ and $\delta$ given by

$$
\begin{aligned}
& \alpha_{0}=\sqrt{c_{33} / \rho}=\alpha_{V}, \beta_{0}=\sqrt{c_{44} / \rho}=\beta_{V}, \\
& \gamma=\frac{c_{66}-c_{44}}{2 c_{44}}, \varepsilon_{T}=\frac{c_{11}-c_{33}}{2 c_{33}}
\end{aligned}
$$

and

$$
\delta=\frac{\left(c_{13}+c_{44}\right)^{2}-\left(c_{33}-c_{44}\right)^{2}}{2 c_{33}\left(c_{33}-c_{44}\right)}
$$

These equations can be used to convert Thomsen parameters to the present representation of VTI parameters. From equation (49) we obtain $\phi$ from

$$
1+2 \varepsilon_{T}=\frac{c_{11}}{c_{33}}=\frac{\alpha_{H}^{2}}{\alpha_{V}^{2}}=\frac{1}{\phi}
$$

Thus $\alpha_{\mathrm{H}}=\alpha_{\mathrm{V}} / \sqrt{\phi}$. Further (50) gives

$$
\sqrt{\left[\left(\alpha_{0}^{2}-\beta_{0}^{2}\right)\left\{(2 \delta+1) \alpha_{0}^{2}-\beta_{0}^{2}\right\}\right]}-\beta_{0}^{2}=c_{13} / \rho=\frac{F}{\rho}=V_{F}^{2} \text {, say }
$$

Hence we obtain, $\eta=V_{\mathrm{F}}^{2} /\left(\alpha_{\mathrm{H}}^{2}-2 \beta_{\mathrm{V}}^{2}\right)$, where $\mathrm{V}_{\mathrm{F}}^{2}$ is given by left hand side of (51).

Xiao et al. [2004] compared four reflection-traveltime inversion methods to get VTI subsurface structure on synthetic data based on a 4-layered structure shown in Table 3, where the VTI parameters of Xiao et al. [2004] has been converted to the present representation; approximate density values have been considered. Figure 5 a shows the phase velocities $\left(c_{\mathrm{VTI}}\right)$ and group velocities $\left(U_{\mathrm{VTI}}\right)$ of fundamental mode Rayleigh waves for this model (Table 3). During computation complex $r_{1}$ and $r_{2}$ appear at least in one of the lower layers even up to period $10 \mathrm{~s}$. We obtain phase velocities $\left(c_{\text {ISO }}\right)$ and group velocities $\left(U_{\text {ISO }}\right)$ for an isotropic model by con- sidering $\phi=\eta=1$ in Table 3. The difference $\left(c_{\mathrm{ISO}}-c_{\mathrm{VTI}}\right)$ is as large as $0.43 \mathrm{~s}$ around the period $1 \mathrm{~s}$ (Figure $5 \mathrm{~b}$ ). Even at a very low period of $0.1 \mathrm{~s}$, this difference is 0.42 s. The difference $\left(U_{\text {ISO }}-U_{\text {VTI }}\right)$ is as large as 0.57 around the period $1.25 \mathrm{~s}$. When period tends to zero, the phase velocity tends to that of the top layer as a half space. The phase velocity top VTI layer as a half space is 0.899 $\mathrm{km} / \mathrm{s}$ using the dispersion equation of Stoneley [1963] and Vinh and Ogden [2004]; velocity of such a half space decreases as $S_{3}^{2}$ decreases. While considering the top layer as an isotropic half space $(\phi=\eta=1)$, we have phase velocity as $0.9194 * \beta_{\mathrm{V}}=0.9194 * 1.4 \mathrm{~km} / \mathrm{s}=1.287$ $\mathrm{km} / \mathrm{s}$ [Ewing et al. 1957]. Thus the difference between Rayleigh wave velocities of the two half spaces are $0.388 \mathrm{~km} / \mathrm{s}$. Grechka et al. [2002] noted that the reflection data cannot be uniquely inverted for VTI parameters without additional information. The large velocity difference in Figure $5 b$ shows that short period phase and group velocities can be used to improve the result of anisotropy in subsurface obtained through anisotropic travel time inversion and NMO velocity analysis. The sensitivities of surface wave velocities to the medium parameters are significant down to about half the wavelength of the wave [Bhattacharya 2015]; the short period range may be considered accordingly.

\begin{tabular}{lcccccc}
\hline Layer No. & $\begin{array}{c}\text { Thickness } \\
(\mathbf{k m})\end{array}$ & $\begin{array}{c}\alpha_{\mathbf{H}} \\
(\mathbf{k m} / \mathbf{s})\end{array}$ & $\begin{array}{c}\beta_{\mathbf{V}} \\
(\mathbf{k m} / \mathbf{s})\end{array}$ & $\phi$ & $\eta$ & $\begin{array}{c}\rho \\
\mathbf{g m} / \mathbf{c m}^{3}\end{array}$ \\
\hline 1 & 0.5 & 3.3130 & 1.400 & 0.7143 & 1.1882 & 2.2 \\
2 & 0.5 & 3.4206 & 1.500 & 0.7692 & 1.1523 & 2.3 \\
3 & 0.5 & 3.5055 & 1.600 & 0.8333 & 1.0780 & 2.4 \\
4 & $\infty$ & 3.7695 & 1.750 & 0.8621 & 1.0395 & 2.5 \\
\hline
\end{tabular}

Table 3. Example of a VTI subsurface [Xiao et al. 2004].

\section{Conclusions}

(a) In a VTI medium, when $S_{3}^{2} \geq 0$, the decay factor $r_{j}(j=1,2)$ is real or imaginary as in a isotropic medium.

(b) In a VTI medium, when $S_{3}^{2}<0$, the decay factor $r_{j}$ becomes complex $(j=1,2)$. This occurrence depends on the medium parameters and c. In the $\phi-\eta$ plane, the complex region of $r_{j}$ is bounded by two nearly parallel lines for given $c / \beta_{\mathrm{V}}$ and $\alpha_{\mathrm{H}} / \beta_{\mathrm{V}}$ (Figure 1). The area of this region decreases with increase of $c / \beta_{\mathrm{V}}$, or $\alpha_{\mathrm{H}} / \beta_{\mathrm{V}}$. We find that $S_{3}^{2}<0$ only if $c<\beta_{\mathrm{V}}$.

(c) We obtain a layer matrix and a delta layer matrix with real elements when $r_{j}$ is real or imaginary. When $r_{j}$ is complex, $r_{1}$ and $r_{2}$ are complex conjugates; using this property, we get these matrices with real elements.

(d) Using T-H method with reduced delta matrix, 
we obtain Rayleigh dispersion equation (47) with real terms in a layered VTI half space.

(e) We evaluate Rayleigh wave phase and group velocities for oceanic and continental VTI models through dispersion equation (47) (Figures 2, 3 and 5). Surface ellipticity has been evaluated from equation (48) (Figure 4).

(f) The dispersion equation (47) contains real terms as in isotropic case. Thus for inversion of observed surface waves to get VTI structure, it may not be necessary to simplify the structure assuming $\phi=\eta=1$ to compute dispersion curves of Rayleigh waves with isotropic codes as has been done in a few studies.

(g) Strong VTI is seen in subsurface particularly with organic rich shales. Such subsurface shows significant difference between dispersion curves of Rayleigh waves for VTI and isotropic subsurface (Figure 5). Thus short period surface waves dispersion data can be used to improve the VTI parameters of the subsurface in conjunction with reflection studies in exploration seismology.

\section{References}

Alkhalifah T., I. Tsvankin (1995) Velocity analysis for transversely isotropic media, Geophysics, 60, 15501566, doi: 10.1190/1.1443888.

Anderson D.L. (1961). Elastic wave propagation in layered anisotropic media, J. Geophys. Res., 66, 29532963.

Anderson D.L. (1962). Love wave dispersion in heterogeneous anisotropic media, Geophysics, 27, 445454.

Anderson D.L. (1965). Recent evidence concerning the structure and composition of the Earth's mantle, in Physics and Chemistry of the Earth, Vol. 6, Pergamum Press, Oxford, pp. 1-131.

Auer L., T.W. Becker, L. Boschi and N. Schmerr (2015). Thermal structure, radial anisotropy and dynamics of oceanic boundary layers, Geophys. Res. Let., 42, 9740-9749, doi: 10.1002/2015GL066246.

Babuska V. and M. Cara (1991). Seismic Anisotropy of the Earth, Modern approaches in geophysics, v. 10, Kluwer Academic Publishers, Dordrecht, The Netherlands, Boston.

Bhattacharya S.N. (1987). Reduction of deep layers in surface wave computation, Geophys. J. R. Astr. Soc., 88, 97-109.

Bhattacharya S.N. and S. Arora (1997). A flattening transformation for P-SV waves in a transversely isotropic earth, Bull. Seismol. Soc. Am., 87, 1297-
1304.

Bhattacharya S.N. (2015). Sensitivities of surface wave velocities to the medium parameters in a radially anisotropic spherical earth and inversion strategies, Annals of Geophysics, 58, No. 5, S0545; doi: 10.4401 / ag-6806.

Buchen, P.W. and R. Ben-Hardor (1997). Free mode surface wave computations, Geophys. J. Int., 124, 869887.

Chen X. (1993). A systematic and efficient method of computing normal modes for multi-layered half space, Geophys. J. Int., 115, 391-409.

Dal Moro G (2014) Surface wave analysis for near surface applications, Elsevier, ISBN 978-0-12-800770-9.

Dunkin J. (1965). Computation of modal solutions in layered, elastic media at high frequencies, Bull. Seismol. Soc. Am., 55, 335-358.

Ewing W.M., W.S. Jardetzky and F. Press (1957). Elastic Waves in Layered Media, McGraw-Hill Book Co. Inc.

Grechka V., I. Tsvankin, A. Bakulin, J.O. Hansen and C. Signer (2002). Joint inversion of PP and PS reflection data for VTI media: A North Sea case study, Geophysics, 67, 1382-1395, doi: 10.1190/1.1512784.

Harkrider D.G. (1964). Surface waves in multi-layered elastic media. I. Rayleigh and Love waves from buried sources in a multi-layered elastic half space, Bull. Seismol. Soc. Am., 54, 627-679.

Harkrider D.G. (1970). Surface waves in multi-layered elastic media. II. Higher mode spectra and spectral ratios from point sources in plane layered earth models, Bull. Seismol. Soc. Am., 60, 1937-1987.

Harkrider D.G. and D.L. Anderson (1962). Computation of surface wave dispersion for multi-layered anisotropic media, Bull. Seismol. Soc. Am., 52, 321332.

Haskell N.A. (1953). Dispersion of surface waves on multi-layered media, Bull. Seismol. Soc. Am., 43, 1734

Huang H., H. Yao and R.D. van der Hilst (2010). Radial anisotropy in the crust of SE Tibet and SW China from ambient noise interferometry, Geophys. Res. Lett., 37, doi: 10:1029/ 2010GL044981.

Ikeda T and T. Matsuoka (2013). Computation of Rayleigh waves on transversely isotropic media by reduced Delta matrix method, Bull. Seismol. Soc. Am., 103, 2083-2093.

Ke G., H. Dong, A. Kristensen and M. Thompson (2011). Modified Thomson-Haskell matrix methods for surface wave dispersion curve calculation and the accelerated root-searching schemes, Bull. Seismol. Soc. Am., 101, 1692-1703. 
Kennett B.L.N. (1974). Reflection, rays and reverberations, Geophys. J. R. Astr. Soc., 64, 1685-1696.

Kennett B.L.N. (1983). Seismic Wave Propagation in Stratified Media, Cambridge Univ. Press, New York

Khan A., L. Boschi, and J.A.D. Connolly (2011). Mapping the Earth's thermochemical structure using global surface wave data, J. Geophys. Res., 116, B01301, doi: 10.1029/ 2010JB007828.

Kustowski B., G. Ekstrom, and A.M. Dziewonski (2008). Anisotropic shear-wave velocity structure of the Earth's mantle: A global model, J. Geophys. Res., 113, doi: 10.1029/ 2007JB005169.

Levin F.K. (1979). Seismic velocities in transversely isotropic media, Geophysics, 44:918-936, doi: $10.1190 / 1.1440985$.

Love A.E.H. (1927). A Treatise on the Mathematical Theory of Elasticity, Cambridge Univ. Press, Cambridge.

Luco J.E. and R.J. Apsel (1983). On the Green's function for a layered half-space, part I, Bull. Seismol. Soc. Am., 73:908-929.

Moschetti M.P., M.H. Ritzwoller, F. Lin and Y. Yang (2010). Seismic evidence for widespread western-US deep-crustal deformation caused by extension, $\mathrm{Na}$ ture, 464:885-889.

Nishimura C.E. and D.W. Forsyth (1989). The anisotropic structure of the upper mantle in the $\mathrm{Pa}$ cific, Geophys. J. R. Astro. Soc., 96, 203-229.

Pei D., J.N. Louie, S.K. Pullammanappallil (2008). Improvements on computation of phase velocity of Rayleigh wave based on the generalized R/T coefficient method, Bull. Seismol. Soc. Am. 98, 280-287.

Raykova R.B. and S.B. Nikolova (2003). Anisotropy in the Earth's crust and uppermost mantle in southeastern Europe obtained from Rayleigh and Love surface waves, J. Appl. Geophys., 54, 247-256.

Sengupta M., K. Katahar, N. Smith, M. Kittridge and J.P. Blangy (2015). Modelling anisotropic elasticity in an unconventional reservoir, The Leading Edge, 34, 1332-1334.

Shapiro N.M., M.H. Ritzwoller, P. Molnar and V. Levin (2004). Thinning and flow of Tibetan crust constrained by anisotropy, Science 305, 233-236.

Socco L.V., S. Foti and D. Boiero (2010). Surface-wave analysis for building near-surface velocity models Established approaches and new perspectives, Geophysics, 75, P75A83-75A102, doi: $10.1190 / 1.3479491$.

Stoneley P. (1963). The propagation of surface waves in an elastic medium with orthorhombic symmetry, Geophysics, 8, 176-186.

Takeuchi H. and M. Saito (1972). Seismic surface waves,
Methods in Computational Physics, Vol. 11, Academic Press, New York, 217-295.

Thomsen L. (1986). Weak elastic anisotropy, Geophysics, 51, 1954-1966. doi: 10.1190/1. 1443818.

Thrower E.N. (1965). The computation of dispersion of elastic waves in layered media, J. Sound. Vib., 2, 210-226.

Tsvankin I. and L. Thomsen (1995). Inversion of reflection traveltimes for transverse isotropy, Geophysics, 60, 1095-1107, doi: 10.1190/1.1443838.

Vinh P.C., and R.W. Ogden (2004). Formulas for Rayleigh wave speed in orthotropic elastic solids, Arch Mech 56, 247-265.

Watson T.H. (1970). A note on fast computation of Rayleigh wave dispersion in a multi-layered halfspace, Bull Seismol Soc Am, 60,161-166.

Xiao C, J.C. Bancroft, R.J. Brown (2004). Estimating anisotropy in layered VTI media, CREWES Research Report 16,1-9.

Xie, J., M.H. Ritzwoller, W. Shen, Y. Yang, Y Zheng, L. Zhou (2013). Crustal anisotropy across Easter Tibet and the Western Yangtze Craton, J Geophys Res, 118, 4226-4252, doi: 10.1002/jgrb.50296.

\footnotetext{
*Corresponding author: Sankar N. Bhattacharya, Lithosphere Study Centre, New Delhi, India.; email: sn_bhattacharya@hotmail.com.

C 2017 by the Istituto Nazionale di Geofisica e Vulcanologia. All rights reserved.
} 